\title{
EDITORIAL
}

\section{Probability and Statistics}

\section{—in Honor of Pao-Lu Hsu's 100th Birthday}

\author{
Dayue CHEN ${ }^{1}, \quad$ Zhi GENG ${ }^{1}, \quad$ Zhi-Ming MA $^{2}$ \\ 1 Department of Probability and Statistics, School of Mathematical Sciences, \\ Peking University, Beijing 100871, China \\ 2 Institute of Applied Mathematics, Academy of Mathematics and Systems Science, \\ Chinese Academy of Sciences, Beijing 100190, China
}

(C) Higher Education Press and Springer-Verlag Berlin Heidelberg 2011

Professor Pao-Lu Hsu (1910-1970) was the pioneer and founding father of Probability and Statistics in China. His research work ranged from limit theory, statistical inference, multivariate analysis, to random matrices and other related topics, and were well recognized by his peers. He was the first Chinese probabilist/statistician with the world fame, though many of his papers were written in Chinese and were published in Chinese journals. He was the first teacher to offer a course in probability and statistics in China. His earlier students included Kai Lai Chung, Shengming Leng, Shouren Wang of the Southwestern United University, Isadore Blumen, Albert Bowker, Erich Lehmann and Ingram Olkin in the USA. In 1956, probability and statistics was enlisted as a key subject to be developed with priority in China. Professor Hsu was appointed as the founding director of the Division of Probability and Statistics established within the Department of Mathematics and Mechanics at Peking University. The division was the very first of the kind in China and served as the seed for a nationwide development subsequently. In particular, he was the mastermind of the intensive training program held at Peking University in 1950s. Many qualified trainees were dispatched to universities all over the country and the curriculum and lecture notes were replicated. For his achievements and contributions, Professor Hsu was elected as a member of the Chinese Academy in 1948 and in 1955. His collected papers were published in Chinese by the Science Press and in English by Springer-Verlag.

It was Professor Hsu's strong wish to promote probability and statistics in China with his best endeavor. He even wanted to subsidize a professional journal with his own money in order to publish papers by young Chinese researchers. Due to many unfortunate reasons, Professor Hsu did not see his plan executed before his untimely death in 1970. Nowadays, probability and statistics has developed very well in China. Almost all probabilists and

\footnotetext{
Received April 8, 2011

E-mail: dayue@math.pku.edu.cn, zgeng@math.pku.edu.cn, mazm@amt.ac.cn
} 
statisticians in China are either Hsu's students or students of his students. The Chinese Society of Probability and Statistics was established in 1981, and the Chinese Journal of Applied Probability and Statistics was launched in 1985, fulfilling a long-time wish of Professor Hsu. Several probabilists were invited to take the floor in the International Congresses of Mathematicians. Chinese statisticians are getting extremely active in the USA and in the world.

To commemorate Professor Pao-Lu Hsu on the occasion of his 100th birthday, the International Conference on Probability and Statistics was held at Peking University in July 2010. 168 probabilists and statisticians from all over the world participated in the conference and 78 distinguished researchers were invited to present their work. Detailed information on the workshop, including the invited speakers, the organizing committee and the conference program, can be found in

http://www.math.pku.edu.cn/misc/probstat/hsu100.htm

We gratefully acknowledge the generous financial support provided by the National Natural Science Foundation of China and Beijing International Center for Mathematical Research.

Dedicated to the memory of late Professor Pao-Lu Hsu, this issue collects some papers on which the presentations were based. All papers in this special issue were refereed. On behalf of the organizing committee, we would like to thank all the participants, speakers, authors, and especially all the referees for their contributions for the success of the conference and publication of this issue.

Probability and Statistics - an International Conference in Honor of Pao-Lu Hsu's 100th Birthday

\section{Program Committee}

Dayue Chen (Peking University)

Zhi Geng (Co-Chair, Peking University)

Shuyuan He (Peking University)

Jun Liu (Harvard University)

Zhi-Ming Ma (Co-Chair, Chinese Academy of Sciences)

Changping Wang (Peking University)

Bin Yu (University of California, Berkeley)

Pingwen Zhang (Peking University) 


\section{Publications of Pao-Lu Hsu}

1. On the limit of a sequence of point sets. Bull Amer Math Soc, 1935, 41: 502-504

2. A note on the indices and numbers of nondegenerate critical points of biharmonic functions (with Kiang Tsai-han). Science Quarterly, Nat Univ Peking, 1935, 5(3): 389-392

3. Contributions to the theory of "Student's" $t$-test as applied to the problem of twosamples. Statist Res Mem, 1938, 2: 1-24

4. On the best unbiased quadratic estimate of the variance. Statist. Res. Mem. 19382 91-104

5. Notes on Hotelling's generalized T. Ann Math Statist, 1938, 9: 231-243

6. A new proof of the joint product moment distribution. Proc Cambridge Philos Soc, 1940, 35: 336-338

7. On the distribution of roots of certain determinantal equations. Ann Eugenics, 1939, 9: $250-258$

8. On $n$-fold iterated limits. J Chinese Math Soc, 1940, 2: 40-63

9. An algebraic derivation of the distribution of rectangular coordinates. Proc Edinburgh Math Soc, 1940, 6(2): 185-189

10. On generalized analysis of variance. Biometrika, 1940, 31: 221-237

11. On the limiting distribution of roots of a determinantal equation. J London Math Soc, 1941, 16: 183-194

12. The limiting distribution of the canonical correlations. Biometrika, 1941, 32: 38-45

13. Analysis of variance from the power function standpoint. Biometrika, 1941, 32: 62-69

14. On the problem of rank and the limiting distribution of Fisher's test function. Ann Eugenics, 1941, 11: 39-41

15. Canonical reduction of the general regression problem. Ann Eugenics, 1941, 11: 42-46

16. The limiting distribution of a general class of statistics. Acad Sinica Science Record, 1942, 1: $37-41$

17. Some simple facts about the separation of degrees of freedom in factorial experiments. Sankhyā, 1943, 6: 253-254

18. The approximate distribution of the mean and variance of a sample of independent variables. Ann Math Statist, 1945, 16: 1-29

19. On the approximate distribution of ratios. Ann Math Statist, 1945, 16: 204-210

20. On the power functions for the $E^{2}$-test and the $T^{2}$-test. Ann Math Statist, 1945, 16: $278-286$

21. On a factorization of pseudo-orthogonal matrices. Quart J Math Oxford Ser, 1946, 17: 162-165

22. Sur un théorème de probabilités dénombrables (with Kai-Lai Chung). C R Acad Sci Paris, 1946, 223: 467-469

23. On the asymptotic distribution of certain statistics used in testing the independence between successive observations from a normal population. Ann Math Statist, 1946, 17: $350-354$

24. Complete convergence and the law of large numbers (with H Robbins). Proc Nat Acad Sci USA, 1947, 33: 25-31

25. The limiting distributions of functions of sample means and application to testing hypotheses. In: Proc Berkeley Symp Math Statist Probability. Berkeley and Los Angeles: Univ of California Press, 1949, 359-402

26. Absolute moments and characteristic function. J Chinese Math Soc (New Series), 1951, 1: 257-280 (in English with abstract in Chinese)

27. A lemma on the coefficient of reduction of a sum of independent variables. Acad Sinica Science Record, 1951, 4: 197-200

28. Appendix: The Jacobians of certain matrix transformations useful in multivariate analysis (Based on lectures of P L Hsu at the University of North Carolina, 1947, by Walter L Deemer and Ingram Olkin). Biometrika, 1951, 38: 345-367 
29. On symmetric, orthogonal, and skew-symmetric matrices. Proc Edinburgh Math Soc Ser, 1953, 2: 37-44

30. On characteristic functions which coincide in the neighborhood of zero. Acta Math Sinica (Succeeding J Chinese Math Soc (New Series)), 1954, 4: 21-32 (in Chinese with abstract in English)

31. On a kind of transformations of matrices. Acta Math Sinica, 1955, 5: 333-346 (in Chinese with abstract in English)

32. On a kind of transformations of matrix pairs. Acta Sci Natur Univ Pekinensis, 1955, 1: 1-16 (in Chinese with abstract in English)

33. Simultaneous transformation of a hermitian matrix and asymmetric or skew-symmetric matrix. Acta Sci Natur Univ Pekinensis, 1957, 3: 167-209 (in Chinese with abstract in English)

34. The differentiability of the probability transition function of a purely discontinuous stationary Markov process on the Euclidean space. Acta Sci Natur Univ Pekinensis, 1958, 4: 257-270 (in Chinese with abstract in English)

35. The absolute continuity of the distribution function in the class L. Acta Sci Natur Univ Pekinensis, 1958, 4: 145-150 (in Chinese with abstract in English)

36. An association scheme $M_{3}(6)$ which is not a $L_{3}$-scheme. Acta Math Sinica, 1964, 14 : 177-178 (in Chinese)

37. The limiting distribution of order statistics. Acta Math Sinica, 1964, 14: 694-714 (in Chinese). By pen name "Ban Cheng" as representative for a seminar conducted by Pao-Lu Hsu

38. Partially balanced incomplete block designs. Prog Math, 1964, 7: 240-281 (in Chinese). By pen name "Ban Cheng" as representative for a seminar conducted by Pao-Lu Hsu

39. A general weak limit theorem for independent distributions. Appendix III in Limit Distributions of Sums of Independent Random Variables, by B V Gnedenko and A N Kolmogorov, translated by K L Chung, revised edition. Boston: Addison-Wesley, 1968

40. On the coincidence property of stochastic matrices (with Jiading Chen and Zhongguo Zheng). Acta Sci Natur Univ Pekinensis, 1979, 15: 21-47 (in Chinese with abstract in English)

41. BIB matrices, simple codes and orthogonal codes. In: Selected Papers of Pao-Lu Hsu. Beijing: Science Press, 1981, 211-225 (in Chinese)

42. Selected Papers of Pao-Lu Hsu. Beijing: Science Press, 1981 (in Chinese)

43. Theory of Sampling. Beijing: Peking University Press, 1982 (in Chinese)

44. Pao-Lu Hsu Collected Papers. New York: Springer-Verlag, 1983

45. Studies in Markoff chains with a finite number of states. Chinese Journal of Applied Probability and Statistics, 1990, 6: 342-353 